TITLE:

\title{
First-principles study of bulk ordering and surface segregation in Pt-Rh binary alloys
}

\section{$\operatorname{AUTHOR}(\mathrm{S})$ :}

Yuge, K; Seko, A; Kuwabara, A; Oba, F; Tanaka, I

\section{CITATION:}

Yuge, K ... [et al]. First-principles study of bulk ordering and surface segregation in Pt-Rh binary alloys. PHYSICAL REVIEW B 2006, 74(17): 174202.

ISSUE DATE:

2006-11

URL:

http://hdl.handle.net/2433/39890

RIGHT:

Copyright 2006 American Physical Society 
PHYSICAL REVIEW B 74, 174202 (2006)

\title{
First-principles study of bulk ordering and surface segregation in Pt-Rh binary alloys
}

\author{
Koretaka Yuge, Atsuto Seko, Akihide Kuwabara, Fumiyasu Oba, and Isao Tanaka \\ Department of Materials Science and Engineering, Kyoto University, Sakyo, Kyoto 606-8501, Japan \\ (Received 19 December 2005; revised manuscript received 11 July 2006; published 8 November 2006)
}

\begin{abstract}
The cluster expansion technique in conjunction with first-principles calculations has been applied in Monte Carlo simulations to derive the configurational thermodynamics of the bulk and (111) surface of Pt-Rh alloys. Lattice-dynamics calculations reveal that the vibrational contribution to $\mathrm{Pt}-\mathrm{Rh}$ bulk phase stability is fairly negligible. Calculated short-range-order parameter, ground state, and ordering transition temperature $T_{c}$ of bulk $\mathrm{Pt}_{50} \mathrm{Rh}_{50}$ are in satisfactory agreement with experimental values in the literature. Calculated composition profiles of the (111) surface at $T=1373 \mathrm{~K}$ show the enrichment of $\mathrm{Pt}$ at the top layer and Pt depleted at the second layer for the entire composition, which is in agreement with experimental observations. At low temperatures, a significant difference is found in the temperature dependence of the layer composition profile between $\mathrm{Pt}_{25} \mathrm{Rh}_{75}$ and $\mathrm{Pt}_{50} \mathrm{Rh}_{50}$. While $\mathrm{Pt}$ composition of the $\mathrm{Pt}_{25} \mathrm{Rh}_{75}$ subsurface shows positive temperature dependence, that of $\mathrm{Pt}_{50} \mathrm{Rh}_{50}$ has a minimum at $T \sim 300 \mathrm{~K}$. The former can be qualitatively interpreted by taking account of the on-site energy only. The latter is due to the occurrence of sublayer-confined phase transition from $(\sqrt{3} \times \sqrt{3}) R 30^{\circ}$ order to disorder alloys.
\end{abstract}

DOI: 10.1103/PhysRevB.74.174202 PACS number(s): 81.30.-t, 68.35.-p, 68.35.Md, 68.35.Rh

\section{INTRODUCTION}

Pt-Rh alloys are widely found in technical applications due to their selective catalytic properties for automotive pollution control, ${ }^{1}$ resistance to corrosion, and thermal stability. Thus phase stability of Pt-Rh alloys has been one of the most attractive subjects of experimental and theoretical research. Pt-Rh bulk alloys are known to show a complete mutual solid solution in $A 1$ structure at high temperatures. ${ }^{2}$ However, it has been argued that Pt-Rh alloys favor phaseseparation or form an ordered structure when temperature decreases. For the former, the existence of miscibility gaps at $T \sim 1000 \mathrm{~K}$ has first been proposed by Raub et al.,$^{3}$ though subsequent attempts to observe these miscibility gaps have consistently failed. ${ }^{4-6}$ Later in 1995, first-principles-based study ${ }^{7}$ predicted the $\mathrm{Pt}-\mathrm{Rh}$ to be an ordering system: $\mathrm{Pt}_{50} \mathrm{Rh}_{50}$ alloys form " 40 " ordered structure below $T=210 \mathrm{~K}$. A recent diffuse $\mathrm{x}$-ray scattering study ${ }^{8}$ has confirmed the presence of local order in Pt-47 at. \% Rh at $T=923 \mathrm{~K}$, and measured the position of the diffuse maximum at $1 \frac{1}{2} 0$, which satisfies the corresponding position of predicted structure " 40 ." However, from a theoretical point of view, the effect of lattice vibration has not been examined in the above calculation. On the contrary, the importance of vibrational effects on bulk phase stability has been actively examined both experimentally ${ }^{9-12}$ and theoretically; ${ }^{13-16}$ for instance, lattice vibrational effects can reverse the stability of $\theta$ and $\theta^{\prime}$ phases for the $\mathrm{Al}_{2} \mathrm{Cu}$ system. ${ }^{16}$ Therefore, in order to confirm the phase stability of bulk Pt-Rh, further investigation including vibrational effects is highly desirable.

Meanwhile, the configurational thermodynamics of Pt-Rh surfaces, in particular surface segregation behavior, is well examined not only for their technical applications, but also for the interest from a theoretical point of view; small differences of sublimation enthalpy and atomic size between $\mathrm{Pt}$ and $\mathrm{Rh}$ are both insufficient to predict the segregation behavior. Furthermore, due to the low mixing enthalpy in bulk $\mathrm{Pt}-\mathrm{Rh},{ }^{7}$ ordering tendencies in the surface region are consid- ered to have negligible effects on segregation. This is in contrast to Pt-Ni surfaces, where a strong ordering results in a reversal $\mathrm{Ni}$ segregation to the top layer, despite a negative segregation energy of Pt. ${ }^{17}$ Early experimental studies of the surface segregation of $\mathrm{Pt}-\mathrm{Rh}$ alloys utilized ion scattering and Auger electron spectroscopy (AES) ${ }^{18-21}$ Probing the surface and first subsurface layers, Pt enrichment at the surface layer was revealed. Absolute compositional depth profiles of the first several layers for (111) and (100) surfaces were investigated through time-of-flight (TOF) atom-probe analysis. ${ }^{22,23}$ These studies found the enrichment of Pt at the top layer while depleted Pt at the second layer. Subsequent experimental investigations by low-energy electron diffraction (LEED), low-energy ion scattering (LEIS), scanning tunneling microscopy (STM), and medium-energy ion scattering (MEIS) have confirmed the early studies' statement, and also elucidated the absence of long-range order at the top layer for a wide range of temperature. ${ }^{24-27}$

Theoretical investigations of surface segregation for the Pt-Rh alloy were mostly confined to semiempirical approaches: the tight-binding Ising model (TBIM), ${ }^{28}$ Monte Carlo simulation with the bond model, ${ }^{29}$ and thermodynamic calculation by means of the quasichemical theory ${ }^{30}$ were employed to derive the surface composition profile at high temperatures. First-principles-based investigation was performed by Ruban et al. ${ }^{31}$ using the Green's-function approach. They combined the first-principles calculation with the surface version of cluster expansion techniques for the $\mathrm{Pt}_{25} \mathrm{Rh}_{75}$ alloy. The derived on-site interactions were used in single-site mean-field simulation. The resultant concentration profiles for (111) and (100) surfaces at $1373 \mathrm{~K}$ agree well with experimental data. However, all the ordering or correlation effects, which can be particularly important at low and intermediate temperatures, are neglected in the on-site mean-field calculation. Thus, up to date theoretical understandings of ordering effects on the surface phase stability are lacking.

In the present study, we have combined the first-principles calculation with the cluster expansion technique, which is applied to Monte Carlo statistical simulations in order to 
investigate the ordering effects on the bulk and surface statistical thermodynamics of Pt-Rh alloys. We first investigated Pt-Rh bulk phase stability. The effect of lattice vibration was taken into account within the harmonic approximation, which led to the temperature-dependent effective cluster interactions. Then we investigated the surface phase stability using a recently proposed Monte Carlo technique ${ }^{32}$ based on the grand canonical ensemble.

This paper is organized as follows. In Sec. II, we briefly describe computational schemes of first-principles calculation, the cluster expansion technique for bulk and surfaces, inclusion of the effects of lattice vibration, and their application to Monte Carlo simulations. In Sec. III A, bulk configurational thermodynamics such as ground-state structure, order-disorder phase transition, and short-range order are well examined in order both to confirm the bulk phase stability and to assess the applicability of the present computational schemes to the surfaces. Calculated surface segregation profiles and ordering tendencies are discussed in Sec. III B.

\section{METHODOLOGY}

\section{A. Computational details of first-principles calculations}

The first-principles calculations were carried out using a density-functional theory (DFT) code, the Vienna ab-initio simulation package (VASP). ${ }^{33-35}$ The all-electron Kohn-Sham equations were solved by employing the projector augmented-wave (PAW) method. ${ }^{36}$ The exchange-correlation functional was described by the generalized gradient approximation (GGA) of Perdew-Wang91 form. ${ }^{37}$ Plane-wave cutoff energy was set to $360 \mathrm{eV}$ throughout the calculation. The numerical error was estimated to be of the order of $1 \mathrm{meV} / \mathrm{at}$ by cutoff convergency tests. To deal with the possible convergence problems for metals, the MethfesselPaxton scheme ${ }^{38}$ was used with a smearing parameter $\sigma$ of $0.08 \mathrm{eV}$.

For Pt-Rh bulk phases, geometry optimization for 32atom supercells, constructed by $2 \times 2 \times 2$ expansion of the fcc unit cell, was performed using $k$ points sampled on the basis of the Monkhorst-Pack scheme ${ }^{39}$ with $8 \times 8 \times 8$ grids. Atomic positions were relaxed until the residual forces became less than $1 \mathrm{meV} / \AA$. Through $k$-point convergence tests, the numerical error was estimated at the order of $1 \mathrm{meV} /$ at. Calculated equilibrium lattice constants at $T$ $=0 \mathrm{~K}$ of pure $\mathrm{Pt}$ and $\mathrm{Rh}$ are 3.98 and $3.84 \AA$, respectively, which are both slightly larger than experimental values at room temperature of 3.92 and $3.80 \AA .{ }^{40}$ This overestimation is typical of the GGA. In order to estimate the vibrational effects, we constructed force constant matrices on the basis of the direct method; ${ }^{41}$ Hellmann-Feynman (HF) forces ${ }^{42}$ were obtained by directly moving a chosen atom $i$ from its equilibrium position along direction $\alpha$ by a displacement of $0.02 \AA$. All the symmetrically nonequivalent displacements were considered in the calculations.

In the case of surfaces, geometry optimization for both 42-atom and 63-atom surface slabs (seven layers of the alloy with $17 \AA$ of vacuum thickness) was performed using $k$ points with $4 \times 6 \times 1$ and $4 \times 4 \times 1$. Details of the geometry optimization technique for the surface slabs are described in Sec. II B. The numerical error of surface energy due to the present $k$-point grid was estimated of $2 \mathrm{meV} /$ at. Using $17 \AA$ vacuum layers, the dependence of surface energy of both $\mathrm{Pt}$ and $\mathrm{Rh}$ with respect to the slab thickness converge within $3 \mathrm{meV} /$ at at the seven-layer slab. The above numerical errors in the calculation on phase stability should be rather small due to cancellation of errors when taking energy differences.

\section{B. Cluster expansion of the total energy}

The total energy of the bulk and surface at a specific configuration in the Pt-Rh system is described using the cluster expansion method ${ }^{43,44}$ here. Let us start from the Helmholtz free energy of a system at temperature $T$ described as

$$
F(T)=E_{\mathrm{el}}(T, V)+F_{\mathrm{vib}}(T, V),
$$

where $E_{\mathrm{el}}(T)$ and $F_{\text {vib }}(T)$ denote the electronic and vibrational contribution to the free energy. When thermal expansion effects are factored out, we can rewrite Eq. (1) as

$$
F(T)=E_{\mathrm{el}}\left(T_{0}, V_{0}\right)+F_{\mathrm{vib}}\left(T, V_{0}\right)+F_{\text {therm }}(T, V),
$$

where $T_{0}$ denotes the temperature of $0 \mathrm{~K}, V_{0}$ is the equilibrium volume at $T=0 \mathrm{~K}$, and $F_{\text {therm }}(T, V)$ is the contribution of thermal expansion, induced by lattice vibration, to the free energy. In this section, we focus on the electronic contribution at $T=0 \mathrm{~K}, E_{\mathrm{el}}(T=0 \mathrm{~K}$ ) (hereafter expressed as $E$ ), which can be directly estimated by the first-principles calculation. Treatment of the vibrational contribution, $F_{\text {vib }}\left(T, V_{0}\right)$ and $F_{\text {therm }}(T, V)$, will be discussed in detail in the following section.

In the cluster expansion for bulk Pt-Rh, the total energy $E$ at a specific configuration $\gamma$ can be expanded in terms of the effective cluster interactions (ECIs),

$$
\begin{aligned}
E(\gamma)= & v^{(0)}+v^{(1)}\left\langle\sigma_{i}\right\rangle^{(\gamma)}+\sum_{f} v^{(2, f)}\left\langle\sigma_{i} \sigma_{j}\right\rangle^{(\gamma)} \\
& +\sum_{f} v^{(3, f)}\left\langle\sigma_{i} \sigma_{j} \sigma_{k}\right\rangle^{(\gamma)}+\cdots
\end{aligned}
$$

where $v^{(n, f)}$ denotes the $n$-body effective interaction in cluster type $f$ and $\left\langle\sigma_{i} \sigma_{j} \cdots \sigma_{k}\right\rangle^{(\gamma)}$ the average products of spin variables $\sigma$ over all lattice sites in configuration $\gamma$. Practically, the expansion of Eq. (3) should be truncated up to a finite order in a proper way. In this study on the Pt-Rh binary alloy, spin variables $\sigma_{i}$ were taken to be +1 when the Pt atom occupies a lattice site $i$, and -1 when the Rh atom occupies the site $i$. For a given set of structure $\{m\}$, we can determine the effective interactions $v^{(n, f)}$ by solving the following equation with respect to a set of $v^{(n, f)}: 45$

$$
\sum_{m}\left|E_{\mathrm{DFT}}^{(m)}-\sum_{n, f} v^{(n, f)}\left\langle\sigma_{i} \sigma_{j} \cdots \sigma_{k}\right\rangle^{(m)}\right|^{2}=\min ,
$$

where $E_{\mathrm{DFT}}^{(m)}$ represents the energy of structure $m$ via DFT calculation.

We have calculated the total energies of 15 fcc-based ordered structures: Pt $(F m \overline{3} m), \mathrm{Pt}_{0.75} \mathrm{Rh}_{0.25}$ (I4/mmm, Pm $\overline{3} m$, 


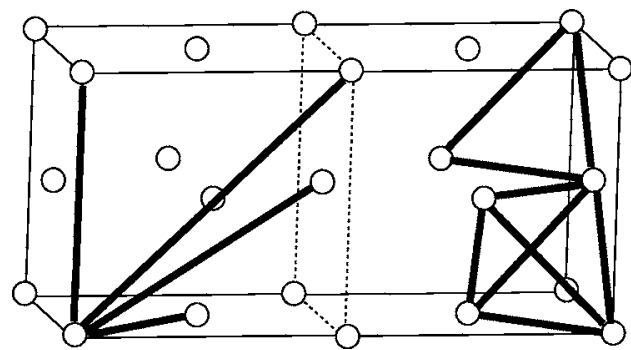

FIG. 1. The four pair, and one triangle and one tetrahedron clusters used in the cluster expansion for bulk Pt-Rh. The clusters are represented by bold lines.

$P 4 / m m m), \mathrm{Pt}_{0.625} \mathrm{Rh}_{0.375}(P 4 / m m m), \mathrm{Pt}_{0.5} \mathrm{Rh}_{0.5}$ (I4 $/$ /amd, $\left.P 4_{2} / m m c, \quad P 4 / n m m, \quad P 4 / m m m, \quad R \overline{3} m\right), \quad \mathrm{Pt}_{0.375} \mathrm{Rh}_{0.625}$ (P4/mmm), $\mathrm{Pt}_{0.25} \mathrm{Rh}_{0.75}$ (I4/mmm, Pm $\left.\overline{3} \mathrm{~m}, \mathrm{P} 4 / \mathrm{mmm}\right)$, and $\mathrm{Rh}(F m \overline{3} m)$. These 15 input structures are based on an optimal set of ordered structures for $\mathrm{CE}$ of the fcc lattice, which Lu et al. suggested, ${ }^{46}$ and additional structures chosen by the present authors to reduce the CV score discussed later. For a given set of inputs, clusters used in the cluster expansion are chosen so that errors of predicted energy using the fitted effective interactions are sufficiently small. We have measured the errors by using the cross-validation $(\mathrm{CV})$ score. ${ }^{47,48}$ While least-squares (LS) errors measure the reproducibility of the known energies of $E_{\mathrm{DFT}}^{(m)}$, the CV score estimates an uncertainty of predicted energies. The $\mathrm{CV}$ score, $\xi$, is given by

$$
\xi^{2}=\frac{1}{N_{s}} \sum_{m=1}^{N_{s}}\left(E_{\mathrm{DFT}}^{(m)}-\hat{E}_{\mathrm{CE}}^{(m)}\right)^{2}
$$

where $N_{s}$ denotes the number of input structures and $\hat{E}_{\mathrm{CE}}^{(m)}$ the energy of structure $m$ predicted by cluster expansion without using $E_{\mathrm{DFT}}^{(m)}$. In the present case, after several trials to choose clusters and additional input structures, we finally obtain the above 15 input structures and multibody clusters as shown in Fig. 1. The resultant $\mathrm{CV}$ score, $\xi$, is $1.2 \mathrm{meV} /$ at and the $\mathrm{LS}$ error is $0.7 \mathrm{meV} /$ at, which is expected to give a sufficiently accurate description of the energetics of bulk ordered structures.

In the case of cluster expansion for surfaces in Pt-Rh, ECIs should become layer-dependent. Therefore, the expression of the bulk cluster expansion of Eq. (3) is rewritten as

$$
\begin{aligned}
E_{\text {surf }}(\gamma)= & v^{(0)}+\sum_{\Lambda}\left[v_{\Lambda}^{(1)}\left\langle\sigma_{\Lambda ; i}\right\rangle^{(\gamma)}+\sum_{\Lambda^{\prime}, f} v_{\Lambda \Lambda^{\prime}}^{(2, f)}\left\langle\sigma_{\Lambda ; i} \sigma_{\Lambda^{\prime} ; j}\right\rangle^{(\gamma)}\right. \\
& \left.+\sum_{\Lambda^{\prime}, \Lambda^{\prime \prime}, f} v_{\Lambda \Lambda^{\prime} \Lambda^{\prime \prime}}^{(3, f)}\left\langle\sigma_{\Lambda ; i} \sigma_{\Lambda^{\prime} ; j} \sigma_{\Lambda^{\prime \prime} ; k}\right\rangle^{(\gamma)}+\cdots\right]
\end{aligned}
$$

where $\Lambda$ specifies the layer index. It is clear that the effective interactions depend not only on the structure type of $f$, but also the layers to which the corresponding cluster belongs. The top two layers are treated as surface, and the rest are treated as bulk: in this case, the eight bulk ECIs split into 18 ECIs for the (111) surface. The resultant cluster type in the (111) surface cluster expansion are shown in Fig. 2.
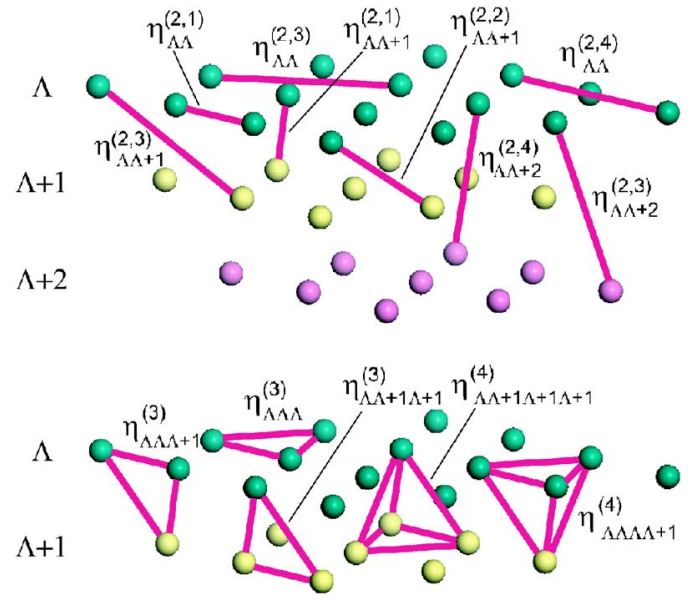

FIG. 2. (Color online) $n$-body clusters, $\eta_{\Lambda \Lambda^{\prime} \ldots}^{(n, f)}$ in type $f$ and in layer $\Lambda, \Lambda^{\prime}, \ldots$, used in the cluster expansion of the (111) surface. $\Lambda$ represents the layer in surface.

A general approach to select input structures for cluster expansion is based on the ground-state search; ${ }^{45}$ starting from ECIs using randomly selected structures, the groundstate search is performed and newly found structures near or on the ground-state line are added to the input structures iteratively. This procedure was indeed successfully applied to bulk systems. However, in the present case of surface cluster expansion, situations are a bit different. Considering the coupling between bulk and surface directly in the surface slab models, the surface ground-state configuration can be defined only for one bulk configuration, i.e., the surface ground state varies when the thermodynamic state of bulk varies. Therefore, the surface ground-state search followed by selection of input surface structures are effective, particularly for a discussion of a surface state in a fixed bulk concentration or configuration. However, we focus here on the surface state in the whole bulk concentration range, including both bulk ordered and disordered structures. It therefore becomes difficult in the present case to determine surface input structures in terms of the ground-state lines. In the present work, we employed an alternative approach based on the concept of sweeping across the correlations of a point cluster, i.e., concentrations: The input surface structures are chosen in terms of a combination of "concentration grid" between surface and bulk parts. The top two layers, described as surface layers, have six atoms in each layer, which makes a layerconcentration grid every $16.67 \%$ for each layer. Consequently, the number of all the combinations of concentration grid for the top two layers becomes 7 times 7, i.e., 49. Considering that each surface slab can have two surfaces, 25 surface slabs are enough to sweep across the surface concentration grid. In addition to the surface layers, we have considered the bulk concentration grid in every $25 \%$. Considering that pure bulk $\mathrm{Pt}$ and $\mathrm{Rh}(0 \%$ and $100 \%)$ should not have binary alloys in the surface, there are three kinds of bulk concentration that couple with the surface concentration grid. Therefore, we first prepare the 3 times 25 plus 2 (pure Pt and $\mathrm{Rh}$ ), i.e., 77 surface slabs for input structures. We have added another 17 structures in order to reduce the CV score, and finally, a total of 94 surface structures are chosen. 
As described in Sec. II A, these 94 surface structures are relaxed through DFT calculations. Therefore, surface relaxation effects, which are quite important for an accurate prediction of surface segregation, are included in the surface ECIs through Eq. (6). In the DFT structure optimization, the in-plane lattice parameter for a surface slab should be fixed to a certain value corresponding to one bulk concentration. In the present case, the bulk concentration corresponds to the concentration in the middle three layers in the surface slab, which is treated as bulk, as described above. Thus we have estimated the in-plane lattice parameters for 94 surface structures by the following: In a similar fashion to expanding total energy given by Eq. (3), we can expand volume in terms of the ECIs. ${ }^{46}$ Therefore, first, we have applied a cluster expansion technique to a volume for bulk Pt-Rh input structures. ECIs are estimated up to a point cluster, which makes a CV score $0.096 \AA^{3}$ in volume for the fcc-unit cell, corresponding to $\sim 0.002 \AA$ in the lattice parameter. Then the resultant ECIs are applied to estimate a volume for the bulklike middle three layers, and we obtain an in-plane lattice parameter corresponding to the bulklike layers by taking a cubic root of volume. This procedure allows us to obtain surface ECIs including changes in the in-plane lattice parameter induced by changes in bulk concentration, i.e., we can treat surface ECIs including different bulk concentration together.

The numerical errors of predicted energy in surfaces are evaluated by the CV score. In the present case, the CV score, $\xi$, is $4.1 \mathrm{meV} /$ at with $\mathrm{LS}$ errors of $3.2 \mathrm{meV} /$ at. These are both slightly larger than the bulk values, but the surface ECIs with errors of this level can still provide reasonable descriptions of the relative energetics of input structures. These bulk and surface ECIs are used in the Monte Carlo simulation as described in the following section.

\section{Effect of lattice vibration}

The effect of lattice vibration is treated using a combination of first-principles lattice dynamics within the harmonic approximation and the cluster expansion in the present study. First, we assess the thermal expansion contribution to the free energy, $F_{\text {therm }}(T, V)$ of Eq. (2), within the simple anharmonic model. ${ }^{49}$ Under this model, $F_{\text {therm }}(T, V)$ can be expressed as ${ }^{50}$

$$
F_{\text {therm }}(T, V)=-\frac{B_{0}}{2 V_{0}}(\Delta V)^{2},
$$

where $B_{0}$ denotes the bulk modulus at $T=0 \mathrm{~K}$, and $\Delta V=V$ $-V_{0}$. Table I shows the calculated bulk modulus $B_{0}$ and equilibrium volume $V_{0}$, experimental values of linear thermal expansion $\varepsilon$ at $T=900 \mathrm{~K}$ measured from $T=0 \mathrm{~K},{ }^{51}$ and the calculated thermal expansion effect $F_{\text {therm }}(T, V)$ using Eq. (7) for $\mathrm{Pt}$ and $\mathrm{Rh}$. The calculated difference of $F_{\text {therm }}(T, V)$ between $\mathrm{Pt}$ and $\mathrm{Rh}$ is less than $1 \mathrm{meV} /$ at at $T=900 \mathrm{~K}$. We have also estimated $F_{\text {therm }}(T, V)$ for the other 11 ordered structures at $\mathrm{Pt}_{0.75} \mathrm{Rh}_{0.25}, \mathrm{Pt}_{0.5} \mathrm{Rh}_{0.5}$, and $\mathrm{Pt}_{0.25} \mathrm{Rh}_{0.75}$ as described in Sec. II $\mathrm{B}$, assuming that the linear thermal expansion $\varepsilon$ of these structures is identical to that of $\mathrm{Pt}$ or $\mathrm{Rh}(\varepsilon=1.007)$. The resultant $F_{\text {therm }}(T, V)$ of ordered structures measured from that of Pt were all less than $1 \mathrm{meV} /$ at at $T=900 \mathrm{~K}$. These
TABLE I. Calculated bulk modulus $B_{0}$ and equilibrium volume $V_{0}$, measured linear thermal expansion $\varepsilon$ at $T=900 \mathrm{~K}$, and calculated thermal expansion effect $F_{\text {therm }}(T, V)$ in $\mathrm{meV} /$ at at $T=900 \mathrm{~K}$.

\begin{tabular}{lcccc}
\hline \hline & $\begin{array}{c}B_{0} \\
(\mathrm{GPa})\end{array}$ & $\begin{array}{c}V_{0} \\
\left(\AA^{3}\right)\end{array}$ & $\varepsilon$ & $\begin{array}{c}F_{\text {therm }}(T, V) \\
(\mathrm{meV} / \mathrm{at})\end{array}$ \\
\hline $\mathrm{Pt}$ & 257.6 & 507.5 & $1.007^{\mathrm{a}}$ & -7.46 \\
$\mathrm{Rh}$ & 250.6 & 454.9 & $1.007^{\mathrm{a}}$ & -6.51 \\
\hline \hline
\end{tabular}

${ }^{\text {aReference } 30 .}$

facts indicate that the thermal expansion effect on free energy should be almost perfectly vanished in Pt-Rh alloys. Therefore for vibrational effects, only the rest term $F_{\text {vib }}\left(T, V_{0}\right)$ [hereafter called just $\left.F_{\text {vib }}(T)\right]$ should be considered.

When a partition function is described in terms of atomic configurations $\gamma$ given by Eq. (A2) in Appendix A, the vibrational contribution can be expanded in a similar way to the electronic contribution $E$ given in Eq. (3), namely,

$$
\begin{aligned}
F_{\mathrm{vib}}(T, \gamma)= & w^{(0)}(T)+w^{(1)}(T)\left\langle\sigma_{i}\right\rangle^{(\gamma)}+\sum_{f} w^{(2, f)}(T)\left\langle\sigma_{i} \sigma_{j}\right\rangle^{(\gamma)} \\
& +\sum_{f} w^{(3, f)}(T)\left\langle\sigma_{i} \sigma_{j} \sigma_{k}\right\rangle^{(\gamma)}+\cdots
\end{aligned}
$$

where $w(T)$ denotes the vibrational contribution to the effective cluster interaction. Note that $w$ depends on temperature $T$, contrary to electronic contribution, $v$. Therefore, in order to include the contribution of lattice vibration, $\{w(T)\}$, the vibrational free energy for a chosen set of structures at temperature $T$ should be estimated.

In the present work, lattice vibration is treated within the harmonic approximation; the vibrational contribution to the Helmholtz free energy at temperature $T, F_{\text {vib }}(T)$, is given by

$$
F_{\mathrm{vib}}(T)=k_{B} T \int_{0}^{\infty} n(\omega) \ln \left\{2 \sinh \left(\frac{\hbar \omega}{2 k_{B} T}\right)\right\} d \omega,
$$

where $K_{B}$ represents the Boltzmann constant, $n(\omega)$ the vibrational density of states (VDOS), and $\omega$ the vibrational angular frequency. We use only diagonal elements of the dynamical matrix ${ }^{52}$ to construct VDOS because in the supercell approach, much larger supercells are required to obtain accurate values of off-diagonal elements than the case of diagonal ones, and thus substantial computational effort is required. ${ }^{53}$ A compatible way to obtain VDOS using only diagonal elements is an effective-medium approximation of the second moment expansion of the Green's function, which has been successfully applied to the vibrational free-energy calculation of the $\mathrm{Fe}-\mathrm{Cu}$ system. ${ }^{50}$ In the effective-medium approximation, VDOS is described as

$$
n_{j \alpha}(\omega)=\frac{4 \omega}{\left(\mu_{j \alpha}^{(1)}\right)^{2} \pi}\left[\left(\mu_{j \alpha}^{(1)}\right)^{2}-\left(\omega^{2}-\mu_{j \alpha}^{(1)}\right)^{2}\right]^{1 / 2},
$$

where $j$ denotes the atom site, $\alpha$ the Cartesian coordinates, and $\mu_{j \alpha}^{(1)}$ corresponds to the diagonal element of the dynamical matrix. In this description of VDOS, vibrational free en$\operatorname{ergy} F_{\text {vib }}$ of Eq. (9) should be rewritten as 


$$
\begin{aligned}
F_{j \alpha} & =k_{B} T \int_{0}^{\infty} n_{j \alpha}(\omega) \ln \left[2 \sinh \left(\frac{\hbar \omega}{2 k_{B} T}\right)\right] d \omega \\
& =\frac{16 k_{B} T}{\pi} \int_{0}^{1} y^{2}\left(1-y^{2}\right)^{1 / 2} \ln \left[2 \sinh \left(\frac{c_{j \alpha} y}{2}\right)\right] d y,
\end{aligned}
$$

where

$$
c_{j \alpha}=\frac{\hbar\left(2 \mu_{j \alpha}^{(1)}\right)^{1 / 2}}{k_{B} T}, \quad y=\frac{\omega}{\left(2 \mu_{j \alpha}^{(1)}\right)^{1 / 2}} .
$$

Therefore, we can estimate the vibrational contribution to the free energy from the $j$ atom in the $\alpha$ direction, $F_{j \alpha}$, from the corresponding diagonal element of the dynamical matrix, which can be obtained through the direct method as described in Sec. II A.

\section{Monte Carlo simulation for bulk Pt-Rh}

In order to obtain the configurational properties of the bulk, Monte Carlo statistical thermodynamic simulations in the canonical ensemble have been carried out on a METROPOLIS algorithm, ${ }^{54}$ using a 16384 -atom cell $(16 \times 16 \times 16$ expansion of the fcc unit cell) under three-dimensional periodic boundary conditions. Including the effect of lattice vibration, the flipping probability from old state $i$ to new state $j$ is chosen as (see Appendix A)

$$
P_{i \rightarrow j}=\exp \left(-\frac{\Delta E_{\mathrm{stat}}^{i \rightarrow j}+\Delta F_{\mathrm{vib}}^{i \rightarrow j}(T)}{k_{B} T}\right),
$$

where $\Delta E_{\text {stat }}^{i \rightarrow j}$ and $\Delta F_{\text {vib }}^{i \rightarrow j}$ represent static and vibrational contributions to the free energy of state $j$ measured from that of state $i$, respectively. A 32000 -atom cell was also used to assess the cell-size dependence of the Monte Carlo results, which found the 16384 atoms to be sufficient for the bulk simulation. A total of 10000 Monte Carlo steps per site were performed for equilibration, followed by 2000 Monte Carlo steps per atom for sampling at each temperature and composition. For the simulation at low temperatures, a simulated annealing algorithm ${ }^{55,56}$ was employed in order to overcome the difficulty in flipping probability: starting at a sufficiently high temperature above the order-disorder transition temperature, the temperature of the simulation box is subsequently decreased by $10 \mathrm{~K}$ after 2000 Monte Carlo steps per site. In each sampling step, the total energy of the system, atomic position, and ordering parameters were stored, and the statistically averaged properties were then obtained.

\section{E. Monte Carlo simulation for Pt-Rh (111) surface}

Since phase equilibrium in the surface region is achieved by the coexistence of the bulk phase, surface Monte Carlo simulation would be carried out in the grand canonical ensemble. A typical procedure to achieve the phase coexistence on the basis of the grand canonical Monte Carlo is to calculate the dependence of composition on chemical potential $\mu$ for a given temperature, which has been previously applied to studies on segregation at surfaces or twist boundaries. ${ }^{57-59}$
However, this technique requires an excessive number of Monte Carlo calculations at each temperature until a trial chemical potential reproduces a desired bulk composition.

In the present study, an alternative approach, direct exchange Monte Carlo (DEMC), ${ }^{32}$ has been adopted, which is based on a grand-canonical ensemble but requires no knowledge of chemical potential in the bulk. The essence of this technique is as follows: A bulk simulation box works only as the reservoir of atoms for a surface slab. Equality of the chemical potentials in both bulk and surface phases is achieved through the transfer of atoms from one box to another, which is based on a Gibbs ensemble Monte Carlo simulation. ${ }^{60}$ Trial energy of transferring atoms for DEMC simulation is given by

$$
\Delta E=\Delta E_{\text {bulk }}(A \rightarrow B)+\Delta E_{\text {surf }}(B \rightarrow A),
$$

where $\Delta E_{\text {bulk }}(A \rightarrow B)$ represents the change in bulk energy by flipping a randomly chosen $A$ atom to $B$, and $\Delta E_{\text {surf }}(B \rightarrow A)$ represents change in energy of the surface by flipping a randomly chosen $B$ atom to $A$. The decision of atom flipping has been made following the trial energy $\Delta E$, and only the atom in the surface slab is changed. This operation should be justified by the fact that bulk is sufficiently large compared to the surface, thus the change in surface has no effects on the thermodynamic state of bulk. Therefore, the equilibrium phase coexistence between bulk and surface can be achieved for a desired bulk composition if the bulk simulation box has been equilibrated beforehand.

The computational procedure is as follows: First, a bulk simulation box of 16384 atoms in a desired composition is equilibrated by a canonical Monte Carlo simulation. Second, surface DEMC starts using the equilibrated bulk box. We have used a surface slab of 6804 atoms $(18 \times 18 \times 21$ layer $)$, which was confirmed to be sufficiently large in terms of the finite-size effect using a larger size surface slab. Similar to the bulk MC calculation, $10000 \mathrm{MC}$ steps per atom for equilibration and $2000 \mathrm{MC}$ steps per atom for sampling were performed at each temperature and bulk composition. For simulations at low temperatures, simulated annealing described above was applied.

\section{RESULTS AND DISCUSSION}

\section{A. Configurational thermodynamics of bulk Pt-Rh}

\section{Ground-state properties}

First, we have calculated formation energies of all possible fcc-based structures consisting of 16 atoms based on an expanding fcc unit cell to investigate the ground-state structures, i.e., structures at $T=0 \mathrm{~K}$. In order to assess lattice vibrational effects on the ground state, we have used two sets of ECIs without and with vibrational contribution, as described in the first (no vib) and second rows [with vib ( $T$ $=0 \mathrm{~K})]$ in Table II. We show in Fig. 3 the resultant groundstate convex hull for the case without vibrational contribution, because with the two sets of ECIs, we have obtained the same ground-state ordered structures. Circles are predicted energies for possible structures, and the full line represents the ground-state line. The ground-state structures of " 40 " and 
TABLE II. The effective cluster interactions (in meV/at) for bulk PtRh.

\begin{tabular}{ccccccc}
\hline \hline & \multicolumn{9}{c}{ Pairs } & Triplets & $\begin{array}{c}\text { Tetrahedron } \\
v^{(4,1)}\end{array}$ \\
\cline { 2 - 5 } & $v^{(2,1)}$ & $v^{(2,2)}$ & $v^{(2,3)}$ & $v^{(2,4)}$ & $v^{(3,1)}$ & 0.3 \\
No vib & 8.1 & -5.6 & 1.7 & 9.9 & -3.0 & 0.3 \\
With vib $(T=0 \mathrm{~K})$ & 8.3 & -5.7 & 1.6 & 9.6 & -3.0 & -0.5 \\
With vib $(T=900 \mathrm{~K})$ & 10.2 & -4.1 & -1.1 & 8.6 & -2.6 & -0.6 \\
\hline \hline
\end{tabular}

$\mathrm{DO}_{22}$ indicated in Fig. 3 are included in 15 input structures of cluster expansion, but other ground-state structures are not. Therefore, in order to confirm the ground-state structures, we have first evaluated the total energies of the structures on the ground-state line indicated in Fig. 3 through DFT calculations. The DFT energies for these structures are all successfully reproduced within an accuracy of $1 \mathrm{meV} /$ at by ECIs obtained from our input structures. Including these new ground states as input structures of cluster expansion, we have then obtained a set of improved ECIs. However, no significant changes have been found in the improved ECIs: before and after including the new structures, the differences of all ECIs do not exceed $0.1 \mathrm{meV}$. Consequently, all the ground-state structures for the improved ECIs are identical to that for the original ECIs from 15 input structures. Therefore, we have confirmed that the ground-state lines in Fig. 3 are found to be reasonable.

The ground-state structures of " 40 " for $\mathrm{Pt}_{50} \mathrm{Rh}_{50}$ and $D 0_{22}$ for $\mathrm{Pt}_{25} \mathrm{Rh}_{75}$, which are both superlattices along the [210] direction, are in agreement with predictions of both early theoretical $^{7,61}$ (without vibrational effects) and experimental studies. ${ }^{8}$ The left-hand columns of Table III show the calculated formation energies of these ordered structures. The values without vibrational effects, -17.5 and $-21.4 \mathrm{meV} /$ at for $\mathrm{DO}_{22}$ and "40," are in reasonable agreement with firstprinciples results of Ref. $61,-16.8$ and $-24.3 \mathrm{meV} / \mathrm{at}$, where structures are fully relaxed, rather than that of Ref. 7, -25.2 and $-29.5 \mathrm{meV} / \mathrm{at}$, for unrelaxed structures. The small but negative formation energies indicate ordering tendencies of the Pt-Rh alloy rather than phase separation. Furthermore, it

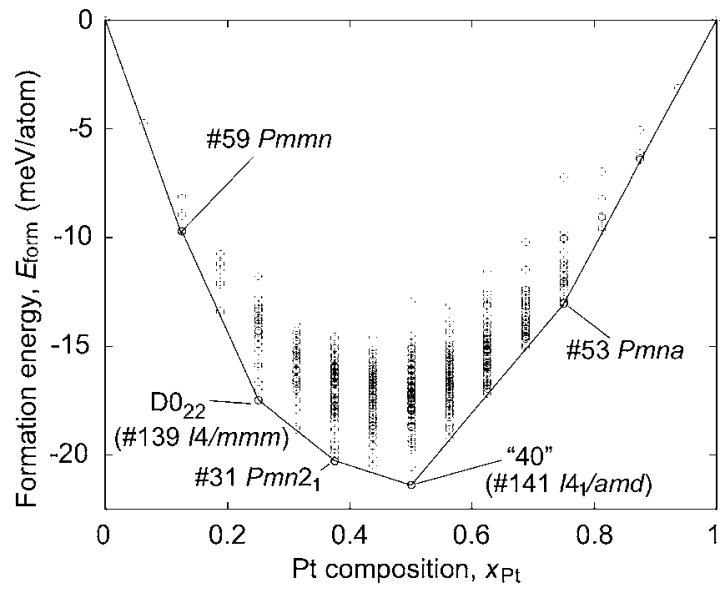

FIG. 3. Ground-state convex hull for Pt-Rh alloys. Circles are predicted energies for possible structures, and the full line represents the ground-state line. is clear from the comparison of values with and without vibrational effects that the contribution of lattice vibration at $T=0 \mathrm{~K}$, i.e., the zero-point motions, to the formation energies are 0.7 and $0.2 \mathrm{meV} /$ at for $D 0_{22}$ and " 40 ," which are almost in negligible order. Therefore, the formation of Pt-Rh ordered structures can be interpreted almost by the electronic contribution, not by the vibrational one.

The order-disorder transition temperatures of ground-state structures were estimated by MC simulations: critical temperature $T_{c}$ is determined by the maximum of the temperature derivative of the statistically averaged total energy. Figure 4 shows the calculated configuration energy $E_{\text {config }}$ including the vibrational effects and its temperature derivative $d E_{\text {config }} / d T$ for the $\mathrm{Pt}_{50} \mathrm{Rh}_{50}$ alloy as a function of temperature. The phase transition from " 40 " to disorder occurs around $T \simeq 110-120 \mathrm{~K}$. When the vibrational effects are neglected, the critical temperature became $T \simeq 100-110 \mathrm{~K}$, which indicates that lattice vibration increases the critical temperature slightly. For $\mathrm{Pt}_{25} \mathrm{Rh}_{75}$, we have found critical temperature $T \simeq 70-80 \mathrm{~K}$. Since at these low transition temperatures the diffusion of atoms is too slow to form the ordered phases, the predicted ground states of " 40 " and $D 0_{22}$ would be hardly detected by experiments.

\section{Disordered alloys in Pt-Rh}

First, we have estimated the formation energies of the disordered Pt-Rh alloy at $T=1100 \mathrm{~K}$, with and without considering vibrational effects. The results are given in the two

TABLE III. Formation energies of random $\Delta H_{\text {form }}^{\text {rand }}$ and ordered alloy in $\mathrm{Pt}_{x} \mathrm{Rh}_{1-x}$ (in meV/at). Calculated formation energies for the random alloy are given at $T=1100 \mathrm{~K}$.

\begin{tabular}{|c|c|c|c|c|}
\hline & \multicolumn{2}{|c|}{ Ordered alloy } & \multicolumn{2}{|c|}{ Random alloy } \\
\hline & $\Delta H_{\text {form }}^{D 0_{22}}$ & $\Delta H_{\text {form }}^{40}$ & $\Delta H_{\text {form }}^{\mathrm{rand}}(x=0.25)$ & $\Delta H_{\text {form }}^{\mathrm{rand}}(x=0.5)$ \\
\hline $\begin{array}{l}\text { Present work } \\
\text { (no vib) }\end{array}$ & -17.5 & -21.4 & -12.3 & -14.8 \\
\hline $\begin{array}{l}\text { Present work } \\
\text { (with vib) }\end{array}$ & -16.8 & -21.2 & -15.0 & -25.3 \\
\hline Calc. $^{\mathrm{a}}$ & -16.8 & -24.3 & & \\
\hline Calc. ${ }^{b}$ & -25.2 & -29.5 & & -11.8 \\
\hline Expt. $^{\mathrm{c}}$ & & & -21.3 & -28.4 \\
\hline
\end{tabular}

Reference 61: US-LDA calculation at $T=0 \mathrm{~K}$.

${ }^{b}$ Reference 7: Cluster expansion with FP-LAPW calculation at $T$ $=0 \mathrm{~K}$ for ordered, and $T \rightarrow \infty$ (no vib) for random alloy.

${ }^{\mathrm{c}}$ Reference 62: Pseudosubregular model with measured activity at $T=1100 \mathrm{~K}$. 


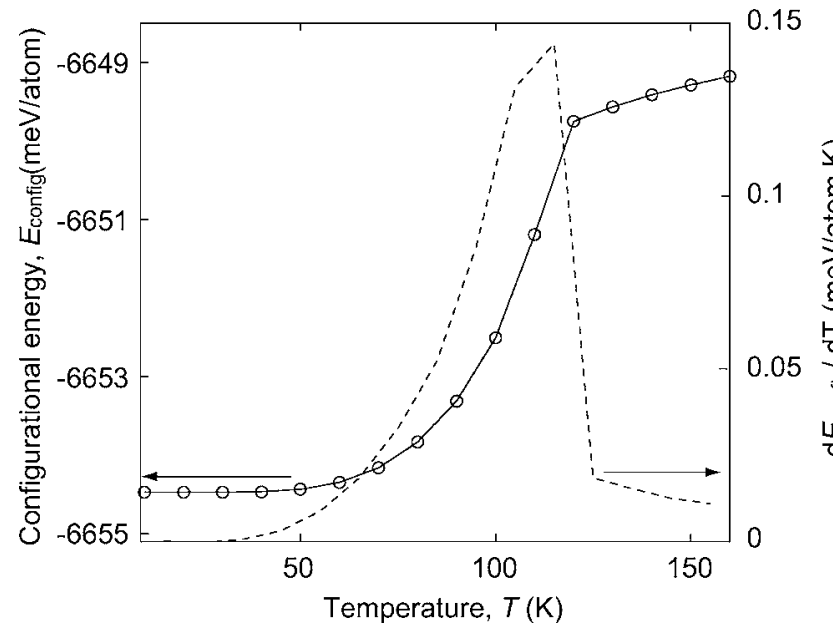

FIG. 4. Calculated configuration energy $E_{\text {config }}$ (open circles) and its temperature derivative $d E_{\text {config }} / d T$ (dashed line) as a function of temperature $T$ for the $\mathrm{Pt}_{50} \mathrm{Rh}_{50}$ alloy.

right-hand columns in Table III with available theoretical ${ }^{7}$ and experimental ${ }^{62}$ data. For the $\mathrm{Pt}_{50} \mathrm{Rh}_{50}$ random alloy, the formation energy without vibrational effects is close to that previously reported. ${ }^{7}$ With vibrational effects, an explicit decrease of $10.5 \mathrm{meV} /$ at is recognized. The resultant formation energy of $-25.3 \mathrm{meV} / \mathrm{at}$ is in good agreement with the experimental value of $-28.4 \mathrm{meV} /$ at. In the case of $\mathrm{Pt}_{25} \mathrm{Rh}_{75}$, vibrational effects also decrease the formation energy, but the amount is smaller, $2.7 \mathrm{meV} /$ at. The large vibrational contributions to the formation energies of the disordered Pt-Rh alloys come from large changes of vibrational free energies themselves for input ordered structures that are used in the cluster expansion when temperature increases. The vibrational free-energy changes for input structures should affect the corresponding ECIs: in the Pt-Rh system, a nonnegligible vibrational contribution clearly appears for particular pair cluster interactions $v^{(2, f)}(f=1, \ldots, 4)$, as is seen in Table II. Positive vibrational contribution to $v^{(2,1)}$ and $v^{(2,2)}$ at $T=900 \mathrm{~K}$ indicates that vibrational free energy decreases when $\mathrm{Pt}$ and $\mathrm{Rh}$ atoms are mixed randomly, while we see a negative contribution to $v^{(2,3)}$ and $v^{(2,4)}$ indicating an increase of vibrational free energy. A significant decrease of formation energies at $x=0.25$ and 0.5 due to lattice vibration therefore mainly comes from the large changes in pair cluster interactions, but cannot be simply interpreted because of competition between the above-mentioned positive and negative vibrational contribution in the same order.

Following the previous section, predicted ordered structures are difficult to detect experimentally due to the relatively low transition temperatures. However, since ordering tendency still remains at finite temperature in a random alloy, comparison of theoretical order parameters and available experimental ones should be quite important in order to assess the impact of alloy ordering. We have calculated the diffuse X-ray scattering intensity and Warren-Cowley SRO parameters $^{63}$ at $T=900 \mathrm{~K}$ using ECIs with and without the vibrational effects (see Table II) for the $\mathrm{Pt}_{50} \mathrm{Rh}_{50}$ alloy. We have found that with and without vibrational effects, calculated maximum intensity appears at $W=1 \frac{1}{2} 0$ point, which

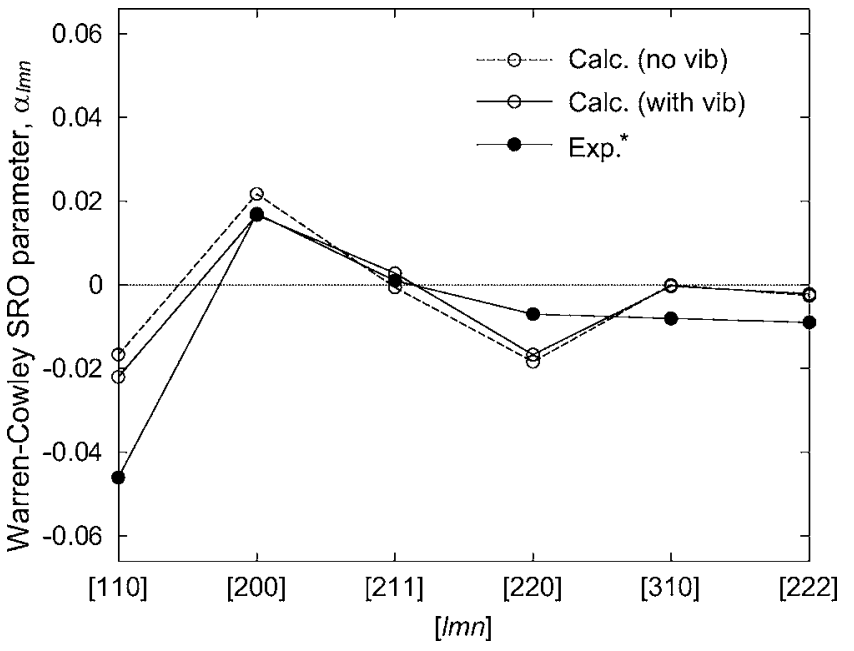

FIG. 5. Warren-Cowley short-range-order parameters. Calculated values in the present work are obtained for $\mathrm{Pt}_{50} \mathrm{Rh}_{50}$ at $T$ $=900 \mathrm{~K}$. Experimental values are for $\mathrm{Pt}_{47} \mathrm{Rh}_{53}$ at $T=923 \mathrm{~K}$, followed by a subsequent quench. [Line with solid circles indicates diffuse x-ray scattering study (Ref. 8)].

agrees with an experimental report. ${ }^{8}$ The results for WarrenCowley SRO parameters are shown in Fig. 5 in comparison with experimental values previously reported. ${ }^{8}$ Calculated and experimental values are in reasonable agreement with each other. From Fig. 5, the lattice vibration slightly increases the ordering and clustering tendency along [110] and [211], while it decreases the clustering tendency along [200]. These ordering and clustering tendencies can be intuitively interpreted by the associated increase of $v^{(2,1)}$ and $v^{(2,2)}$ and the decrease of $v^{(2,3)}$ when including the vibrational effects as shown in Table II. However, the features of local ordering are unchanged even when including the vibrational effects. To summarize, the vibrational effect on Pt-Rh phase stability is almost negligible in particular at low temperatures; predicted ordered structures, their formation energy, and critical temperature are satisfactorily estimated without vibrational effects. For disordered alloys at high temperatures, the formation energies of random alloys are slightly affected by the lattice vibration, yet their SRO parameters are reasonably reproduced without considering the vibrational effects. Therefore, we conclude that the bulk phase stability can be successfully predicted by the temperature-independent effective cluster interactions, $\{v\}$.

\section{B. Surface segregation and ordering in Pt-Rh (111)}

\section{Surface segregation profiles at high temperatures}

Surface segregation behavior in a phase-separating binary system can be uniquely predicted by the difference in the surface energy of pure constituent systems, since mixing of alloy components always results in positive energy costs; the alloy component with the lower surface energy is expected to segregate to the surface. On the contrary, in an ordering system, concentration profiles are indeed determined by the competition between ordering and segregation tendencies. Following the previous discussion, Pt-Rh is labeled as an 
TABLE IV. Effective cluster interactions for the Pt-Rh (111) surface and bulk counterparts (in meV/at).

\begin{tabular}{|c|c|c|c|}
\hline & \multicolumn{3}{|c|}{$\Lambda$} \\
\hline & 1 & 2 & 3 (bulk) \\
\hline $2\left(v_{\Lambda}^{(1)}-v_{\text {bulk }}^{(1)}\right)$ & -335.8 & 110.6 & 0 \\
\hline$v_{\Lambda \Lambda}^{(2,1)}$ & 3.9 & 6.2 & 4.1 \\
\hline$v_{\Lambda \Lambda+1}^{(2,1)}$ & -6.4 & 2.0 & 2.0 \\
\hline$v_{\Lambda \Lambda+1}^{(2,2)}$ & -4.3 & -2.8 & -2.8 \\
\hline$v_{\Lambda \Lambda}^{\Lambda \Lambda+1}$ & 4.4 & -15.5 & 0.4 \\
\hline$v_{\Lambda \Lambda+1}^{(2,3)}$ & 6.1 & 0.4 & 0.4 \\
\hline$v_{\Lambda \Lambda+2}^{(2,3)}$ & 0.2 & 0.2 & 0.2 \\
\hline$v_{\Lambda \Lambda}^{(2,4)}$ & 9.4 & 23.0 & 5.0 \\
\hline$v_{\Lambda \Lambda+2}^{(2,4)}$ & 2.5 & 2.5 & 2.5 \\
\hline$v_{\Lambda \Lambda \Lambda}^{(3)}$ & -6.8 & -1.7 & -0.8 \\
\hline$v_{\Lambda \Lambda \Lambda+1}^{(3)}$ & -3.1 & -0.6 & -0.6 \\
\hline$v_{\Lambda \Lambda+1 \Lambda+1}^{(3)}$ & 0.2 & -0.6 & -0.6 \\
\hline$v_{\Lambda \Lambda \Lambda \Lambda+1}^{(4)}$ & 4.4 & 0.1 & 0.1 \\
\hline$v_{\Lambda \Lambda+1 \Lambda+1 \Lambda+1}^{(4)}$ & 2.4 & 0.1 & 0.1 \\
\hline
\end{tabular}

ordering system, yet its ordering tendencies are relatively small. Thus, at a first insight, surface segregation behavior at sufficiently high temperatures should be roughly predicted by the surface energy difference between Pt and Rh, defined as

$$
\Delta E_{\mathrm{surf}}=E_{\mathrm{surf}}^{\mathrm{Pt}}-E_{\mathrm{surf}}^{\mathrm{Rh}},
$$

where $E_{\text {surf }}^{\mathrm{Pt}}$ and $E_{\text {surf }}^{\mathrm{Rh}}$ represent the surface energies of $\mathrm{Pt}$ and $\mathrm{Rh}$, respectively. Present calculation estimated $\Delta E_{\text {surf }}$ of $-0.40 \mathrm{~J} / \mathrm{m}^{2}$, where early $a b$ initio calculations ${ }^{64-66}$ estimated $-0.43,-0.17$, and $-0.34 \mathrm{~J} / \mathrm{m}^{2}$, respectively, and experiment $^{67}$ showed $-0.22 \mathrm{~J} / \mathrm{m}^{2}$. The negative sign of $\Delta E_{\text {surf }}$ indicates the lower surface energy of $\mathrm{Pt}$ than that of $\mathrm{Rh}$, which leads to the pronounced Pt segregation to the surface. However, since the first several or more layers would contribute to the surface energy, layer-dependent concentration profiles cannot be estimated only by the surface energy difference.

Further quantitative estimation of surface segregation tendencies can be achieved by comparison of the calculated on-site energies $v^{(1)}$ between surface and bulk, which is made in Table IV along with other effective cluster interactions. Note that ECIs in Table IV do not contain the effects of lattice vibration. Twice the on-site energy differences, $2\left(v_{\Lambda}^{(1)}-v_{\text {bulk }}^{(1)}\right)$ in Table IV, represents the on-site energy cost of flipping the Pt atom in bulk and Rh in surface layer $\Lambda$. Therefore, the negative sign of the on-site energy differences, $2\left(v_{\Lambda}^{(1)}-v_{\text {bulk }}^{(1)}\right)$, of the top layer $(-335.8 \mathrm{meV})$ favors $\mathrm{Pt}$ segregation to the top layer, while the positive sign of the second layer (110.6 meV) favors Rh segregation. Additionally, from Table IV, pair and higher-order effective interactions are relatively smaller than the on-site energy differ-

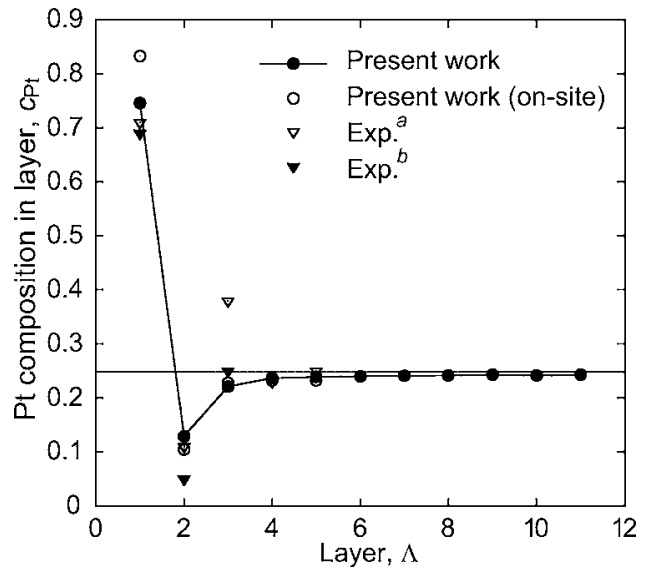

FIG. 6. Layer composition of $\mathrm{Pt}$ for $\mathrm{Pt}_{25} \mathrm{Rh}_{75}$ at $T=1373 \mathrm{~K}$. Closed circles denote the results of calculation including all the ECIs, and open circles those of calculation taking account of only on-site interactions. Open triangle, LEED study ( $T=1373 \mathrm{~K}$ ) (Ref. 25); closed triangle, MEID study ( $T=1300 \mathrm{~K})$ (Ref. 27).

ences. These facts allow us to predict the $\mathrm{Pt}$ and $\mathrm{Rh}$ segregation to the top and second layers, respectively, of the Pt-Rh (111) surface at high temperatures.

Figure 6 shows calculated surface concentration profiles for $\mathrm{Pt}_{25} \mathrm{Rh}_{75}$ at $T=1373 \mathrm{~K}$ using the effective interactions in Table IV and DEMC, in comparison with experimental data previously reported. ${ }^{25,27}$ Closed circles denote the calculated segregation profiles including all the ECIs (hereafter called "full-ECI" calculation), while open circles show those only with the on-site energy interactions. From the comparison of on-site and full-ECI calculation, we found a significant decrease of $\mathrm{Pt}$ and $\mathrm{Rh}$ segregation to the top and the second layer. Calculated $\mathrm{Pt}$ and $\mathrm{Rh}$ concentrations at respective layers are reduced to $c_{\mathrm{Pt}}=0.746$ and $1-c_{\mathrm{Pt}}=c_{\mathrm{Rh}}=0.871$ compared to the results of on-site energy calculation, $c_{\mathrm{Pt}}=0.833$ and $c_{\mathrm{Rh}}=0.896$, which indicates that ordering effects suppress the $\mathrm{Pt}$ and $\mathrm{Rh}$ segregation to the respective layers. Between the calculated and experimental results of Fig. 6, we see a reasonable agreement: (i) $\mathrm{Pt}$ and $\mathrm{Rh}$ segregate to the top and second layer, respectively, and (ii) Pt concentration for deeper than the fourth layer becomes almost identical to that for bulk, indicated by the broken line. For the third layer, calculated results of $c_{\mathrm{Pt}}=0.221$ are in agreement with the MEID (Ref. 27) results of 0.25 rather than the LEED results of 0.38 , which still shows an oscillation of Pt composition.

The dependence of layer-resolved surface Pt concentration $c_{\mathrm{Pt}}^{s}$ on bulk composition $c_{\mathrm{Pt}}^{b}$ has been investigated at $T$ $=1373 \mathrm{~K}$. The results are presented in Fig. 7. It can be clearly seen that for all the bulk composition, the top layer favors $\mathrm{Pt}$ segregation and the second layer Rh segregation, while Pt composition in deeper layers than the third layer becomes rapidly close to that in bulk.

In the above discussion of segregation behavior, the effects of lattice vibration have not been considered. Following Sec. III A, bulk phase stability can be reasonably interpreted only by taking account of the temperature-independent ECIs, $\{v\}$. However, compared to the Pt-Rh bulk, the vibrational effects can be enhanced in the surface region since the vibrational entropy of atoms in the surface region, in particular 


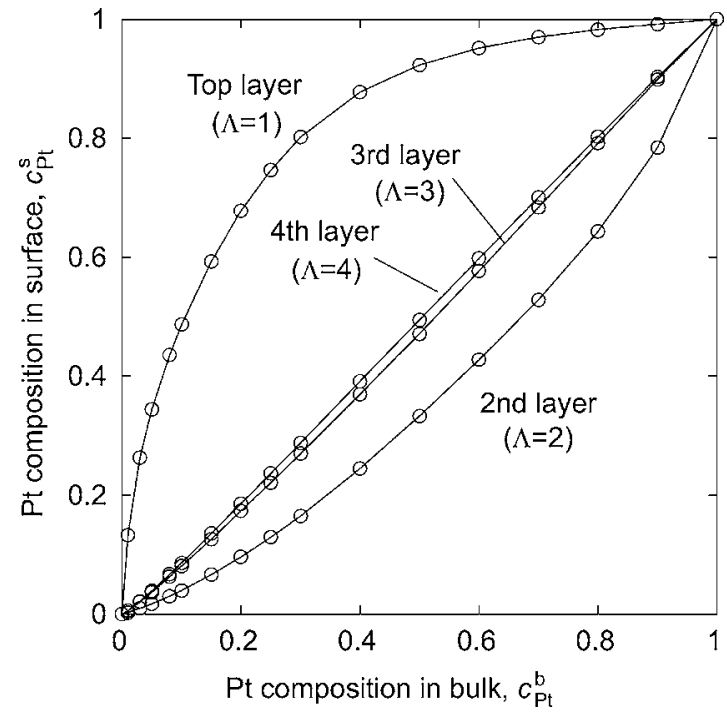

FIG. 7. Calculated surface Pt composition $c_{\mathrm{Pt}}^{\mathrm{s}}$ for the first four layers, as a function of bulk composition $c_{\mathrm{Pt}}^{b}(T=1373 \mathrm{~K})$.

perpendicular to the surface plane, is expected to deviate from that in bulk. Intuitively speaking, alloy components with lower vibrational contribution to surface free energy prefer to segregate to the surface. In the present work, in order to investigate the vibrational effects on surface segregation qualitatively, the vibrational contribution to the on-site energy, $v^{(1)}$, was investigated. In the cluster expansion method, the vibrational contribution to twice the on-site energy difference $2\left(v_{\Lambda}^{(1)}-v_{\text {bulk }}^{(1)}\right)$ in layer $\Lambda, F_{\text {vib }}^{\text {on-site, } \Lambda}$, can be described as

$$
\begin{aligned}
F_{\mathrm{vib}}^{\mathrm{on}-\text { site, } \Lambda}(T)= & 2\left\{\left[F_{\mathrm{vib}}^{\mathrm{Pt}, \Lambda}(T)-F_{\mathrm{vib}}^{\mathrm{Rh}, \Lambda}(T)\right]\right. \\
& \left.-\left[F_{\mathrm{vib}}^{\mathrm{Pt} \text { bulk }}(T)-F_{\mathrm{vib}}^{\mathrm{Rh}, \text { bulk }}(T)\right]\right\},
\end{aligned}
$$

where $F_{\text {vib }}^{A, \Lambda}(T)$ and $F_{\text {vib }}^{A \text {,bulk }}(T)$ denote the vibrational free energy in surface layer $\Lambda$ and that in bulk for alloy component $A$. For the vibrational free energy in surface layer $\Lambda$, we have applied the direct method and Eq. (11) to the estimation of $F_{\text {vib }}^{\mathrm{A}, \Lambda}(T)$. The calculated vibrational contribution $F_{\mathrm{vib}}^{\mathrm{on}-\mathrm{site}, \Lambda}$ for the first two top layers is shown in Fig. 8 as a function of temperature $T$. The results show the negative sign of $F_{\text {vib }}^{\text {on-site }, \Lambda}$ both for the top and second layers, indicating that lattice vibration increases Pt segregation to the first two layers. In particular, at high temperatures, the effects of lattice vibration should not be negligible; for instance, at $T=1373 \mathrm{~K}$, $F_{\text {vib }}^{\text {on-site, } \Lambda}$ is estimated to be -46.5 and $-11.6 \mathrm{meV} /$ at for $\Lambda$ $=1$ and 2 , which are around $11-14 \%$ of $2\left(v_{\Lambda}^{(1)}-v_{\text {bulk }}^{(1)}\right)$, respectively. When we take into account the vibrational contribution only to the on-site energy, the resultant surface concentration for $\mathrm{Pt}_{25} \mathrm{Rh}_{75}$ at $T=1373 \mathrm{~K}$ becomes $c_{\mathrm{Pt}}=0.810$ and $c_{\mathrm{Rh}}=0.863$ for the top and second layers, which deviate by +0.064 and -0.008 from the values without vibrational effects. Meanwhile, with the decrease of temperature, the vibrational contribution $F_{\mathrm{vib}}^{\mathrm{on}-\mathrm{dit}, \Lambda}$ shows an almost linear decrease, and finally it becomes almost zero at $T=0 \mathrm{~K}$, which is in negligible order to $2\left(v_{\Lambda}^{(1)}-v_{\text {bulk }}^{(1)}\right)$.

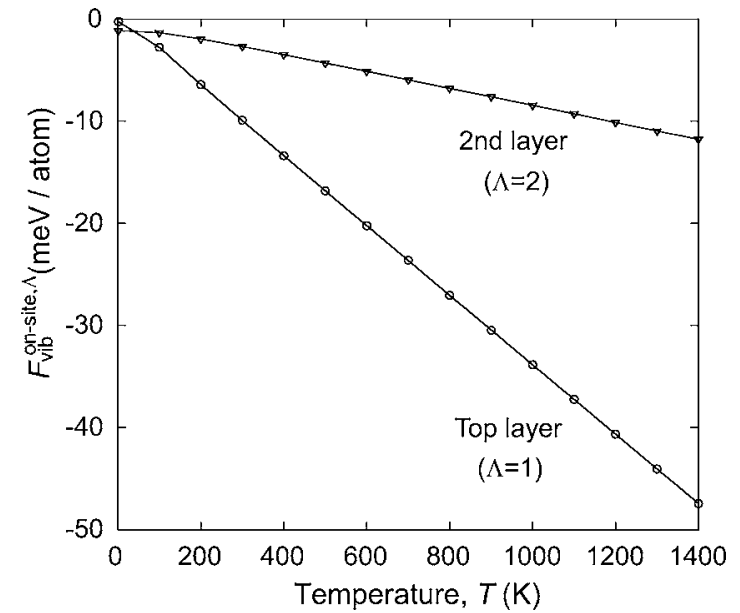

FIG. 8. Calculated vibrational contribution to twice the on-site energy difference in Table IV.

\section{Surface segregation at low temperatures}

In this section, we will extend our discussion to the $\mathrm{Pt}$ segregation behavior at low temperatures. When temperature decreases, segregation tendency to the surface region is expected to enhance due to the decrease of configurational entropy contribution, if all the ordering effects are negligible. Furthermore, from the above discussion, we have seen that the vibrational contribution to the surface thermodynamic state is decreased significantly with the decrease of temperature. Therefore, the effects of the lattice vibration will be neglected in the following discussions.

We have first calculated Pt concentrations of the top two layers using full-ECI and on-site energy, respectively, for $\mathrm{Pt}_{25} \mathrm{Rh}_{75}$ as a function of temperature. The results are given in Fig. 9 along with experimental values. ${ }^{25}$ For all three results in Fig. 9, Pt and Rh segregation to the top and second layers exhibit a monotonous enhancement with a decrease of temperature. Pt and Rh concentration of the top and second layers by the full-ECI calculation (open circles with solid

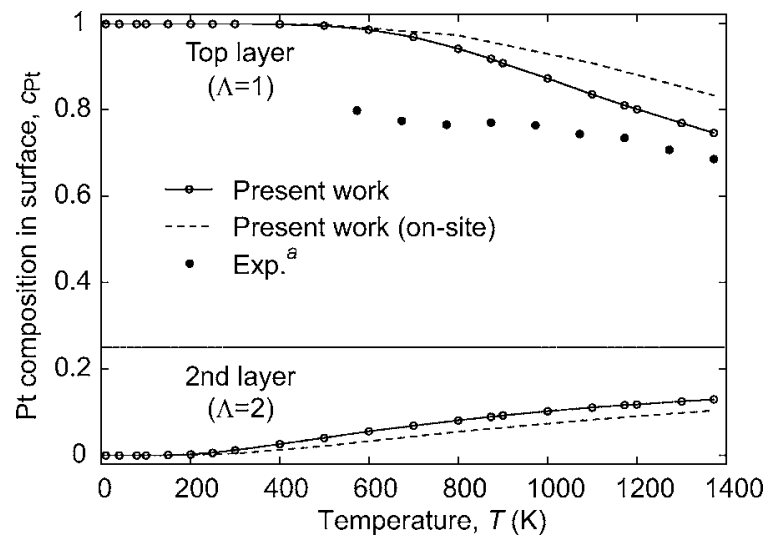

FIG. 9. Temperature dependence of Pt composition of the first two top layers for the $\mathrm{Pt}_{25} \mathrm{Rh}_{75}$ (111) surface. Open circles with solid curves denote the results of calculation including all the ECIs, and dashed lines taking account of only on-site interactions. Solid circles, LEIS study (Ref. 25). 


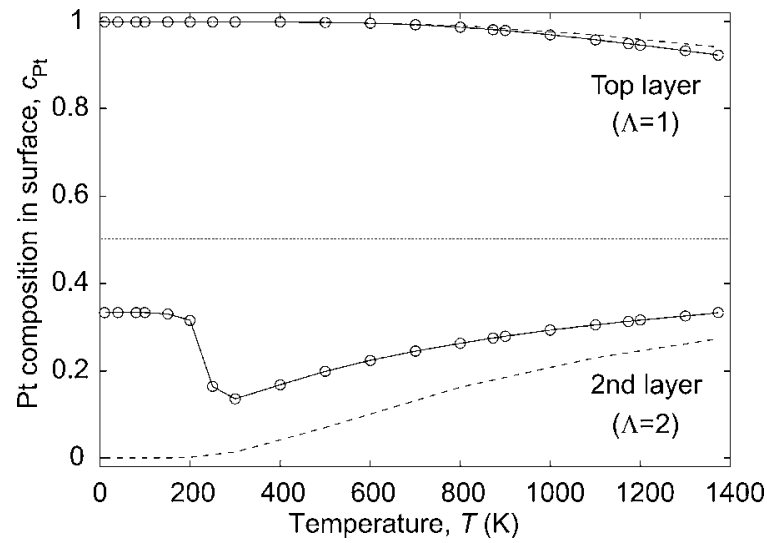

FIG. 10. Temperature dependence of Pt composition of the first two top layers for the $\mathrm{Pt}_{50} \mathrm{Rh}_{50}$ surface. Open circles with solid lines denote the results of calculation including all the ECIs, and dashed lines taking account of only on-site interactions.

lines) is lower than that of the on-site energy (dashed lines) in all the temperature range. Therefore, ordering effects always suppress the segregation of $\mathrm{Pt}$ and $\mathrm{Rh}$. When temperature decreases, calculated Pt concentration of the top layer, $c_{\mathrm{Pt}}$, becomes almost 1.0 around $T=500-600 \mathrm{~K}$, while the experimental one shows $c_{\mathrm{Pt}} \simeq 0.8$ at $T=573 \mathrm{~K}$. Moreover, experimental Pt concentration (closed circles) increases more slowly with the decrease of temperature than that for the full-ECI calculation (open circles). This may be attributed to experimental difficulty in the achievement of equilibrium due to slow diffusion of atoms at low temperatures.

Second, we have calculated Pt concentration of the top and second layers for the $\mathrm{Pt}_{50} \mathrm{Rh}_{50}$ surface (Fig. 10). For the top layer, Pt segregation exhibits monotonous enhancement with the decrease of temperature for both on-site and fullECI calculations, which is a similar behavior to the case of $\mathrm{Pt}_{25} \mathrm{Rh}_{75}$. However, for the second layer, an explicit difference in temperature dependence of Pt concentration can be seen: for full-ECI calculation, Pt segregation shows a minimum around $T=300 \mathrm{~K}$, while for on-site energy calculation, Pt concentration decreases monotonously. This anomalous behavior of the temperature dependence of Pt concentration

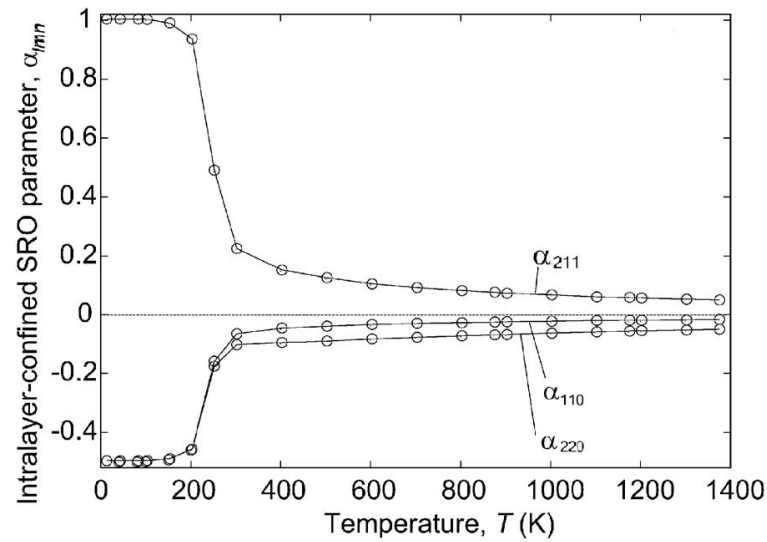

FIG. 11. Calculated intralayer-confined short-range-order (SRO) parameters of the $\mathrm{Pt}_{50} \mathrm{Rh}_{50}$ second layer as a function of temperature.

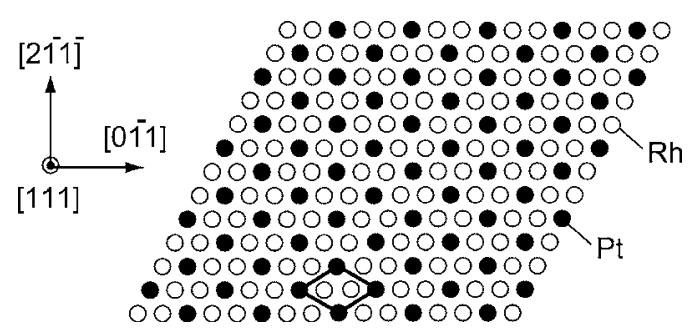

FIG. 12. Simulated $(\sqrt{3} \times \sqrt{3}) R 30^{\circ}$ structure of the second surface layer in $\mathrm{Pt}_{50} \mathrm{Rh}_{50}$ at $T=10 \mathrm{~K}$ viewed from the [111] direction. The closed circles denote Pt atoms, and the open circles Rh atoms. The rhombus framed by bold lines denotes the primitive unit of the ordered structure.

should be due to the ordering effects. In order to investigate this anomalous temperature dependence of the Pt segregation, we have calculated the intralayer-confined short-rangeorder parameters of the $\mathrm{Pt}_{50} \mathrm{Rh}_{50}$ second layer as a function of temperature. The calculated first three intralayer SRO parameters, $\alpha_{110}, \alpha_{211}$, and $\alpha_{220}$, are shown in Fig. 11. Corresponding to the minimum of Pt concentration in Fig. 10, the three SRO parameters exhibit significant change around $T$ $=200-250 \mathrm{~K}$. This can be ascribed by the occurrence of a layer-confined order-disorder phase transition at a critical temperature between 200 and $250 \mathrm{~K}$. The simulated ordered structure at $T=10 \mathrm{~K}$ in the DEMC calculation is illustrated in Fig. 12, whose primitive unit is represented by the rhombus framed by bold lines, which is designated as a $(\sqrt{3} \times \sqrt{3}) R 30^{\circ}$ structure in a composition of $\mathrm{Pt}_{33.3} \mathrm{Rh}_{66.7}$. The predicted ordered structure has a layer-confined superlattice along the [211] direction, which consists of alternating one $\mathrm{Pt}$ and two Rh rows as clearly seen from Fig. 12. In other words, this structure favors like-atom pairs along all the intralayer [211] direction, which satisfies the corresponding negative sign of the effective pair interaction, $v_{\Lambda \Lambda}^{(2,3)}=$ $-5.2(\mathrm{meV})$, in Table IV. However, as shown in Table IV, effective interactions for interlayers and intralayers are really complicated, thus the formation of the $(\sqrt{3} \times \sqrt{3}) R 30^{\circ}$ is difficult to interpret simply in terms of the signs of effective interactions. Table $\mathrm{V}$ summarizes the predicted ground-state structures of the first two surface layers and bulk for $\mathrm{Pt}_{25} \mathrm{Rh}_{75}$ and $\mathrm{Pt}_{50} \mathrm{Rh}_{50}$, and the corresponding simulated structures of the first five top layers for $\mathrm{Pt}_{50} \mathrm{Rh}_{50}$ and $\mathrm{Pt}_{25} \mathrm{Rh}_{75}$ at $T$ $=10 \mathrm{~K}$ are shown in Fig. 13. Since the order a structure $(\sqrt{3} \times \sqrt{3}) R 30^{\circ}$ of the second layer for $\mathrm{Pt}_{50} \mathrm{Rh}_{50}$ has not been reported so far, it should be interesting to perform quantitative experiments of layer-resolved atomic arrangements for this surface.

TABLE V. Predicted ground-state structures of the first two top layers and bulk. Ordered structures are written with their transition temperatures $T_{c}$ in parentheses.

\begin{tabular}{ccc}
\hline \hline & $\mathrm{Pt}_{25} \mathrm{Rh}_{75}$ & $\mathrm{Pt}_{50} \mathrm{Rh}_{50}$ \\
\hline Top layer & $\mathrm{Pt}$ & $\mathrm{Pt}$ \\
Second layer & $\mathrm{Rh}$ & $(\sqrt{3} \times \sqrt{3}) R 30^{\circ}(200-250 \mathrm{~K})$ \\
Bulk & $D 0_{22}(70-80 \mathrm{~K})$ & “40” $(110-120 \mathrm{~K})$ \\
\hline \hline
\end{tabular}



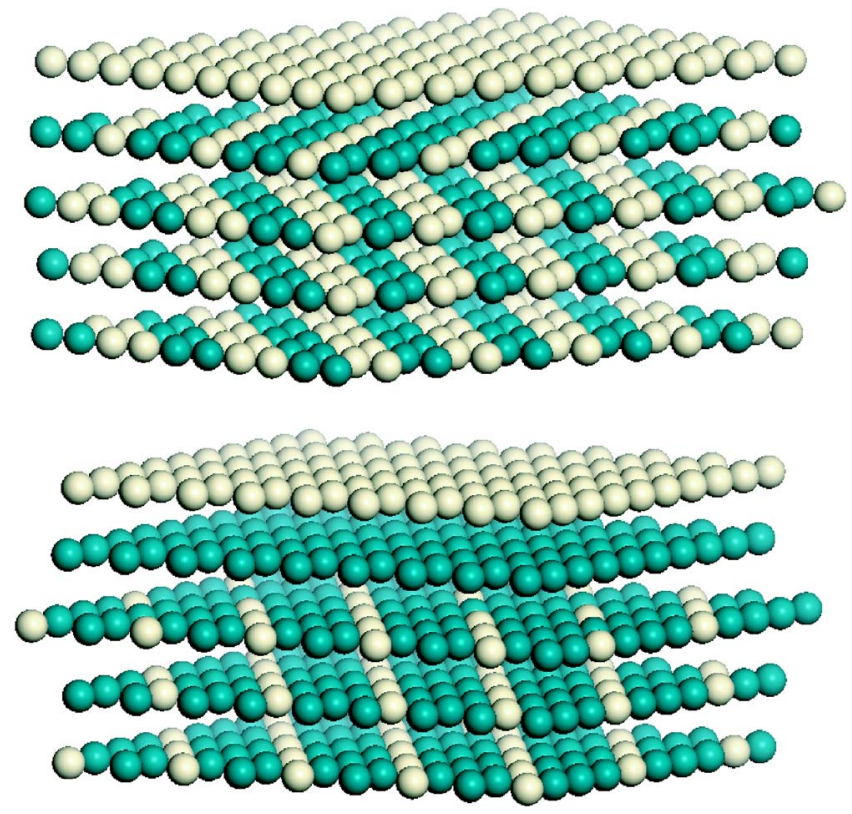

FIG. 13. (Color online) Simulated structures of five top layers of (111) surface in $\mathrm{MC}$ simulations at $T=10 \mathrm{~K}$. The upper figure shows the $\mathrm{Pt}_{50} \mathrm{Rh}_{50}$ alloy, and the lower the $\mathrm{Pt}_{25} \mathrm{Rh}_{75}$. The yellow spheres represent $\mathrm{Pt}$ atoms, and green spheres $\mathrm{Rh}$ atoms.

\section{CONCLUSIONS}

We have investigated the configurational thermodynamics of the bulk and (111) surface of Pt-Rh alloys using the combination of cluster expansion and Monte Carlo statistical simulation, on the basis of the density-functional theory. The first-principles-based lattice-dynamics calculations revealed that (i) thermal expansion effects on bulk phase stability are fairly negligible, (ii) the lattice vibration has no significant effects on formation energies of the ground state, and (iii) the formation energies of Pt-Rh random alloys are significantly decreased by lattice vibration, which became close to the experimental values. Including the vibrational effects, we have confirmed the ground-state order structures of $D_{22}$ and " 40 " for $\mathrm{Pt}_{25} \mathrm{Rh}_{75}$ and $\mathrm{Pt}_{50} \mathrm{Rh}_{50}$, which are in agreement with previous theoretical and experimental predictions. The simulated surface composition profiles at $T=1373 \mathrm{~K}$ show the $\mathrm{Pt}$ enrichment at the top layer while Pt depleted at the second layer for all the composition. Comparison of the on-site energy and full-ECI calculation revealed the ordering effects significantly suppress the $\mathrm{Pt}$ and $\mathrm{Rh}$ segregation to the respective layers. The calculated vibrational contribution to the on-site energy difference between bulk and surface indicates that lattice vibration enhances the Pt segregation to both the top and second layers in particular at high temperatures, while at low temperatures, the vibrational effects on surface segregation become negligible. The calculated surface composition showed a monotonous increase with increasing temperature for $\mathrm{Pt}$ and $\mathrm{Rh}$ segregation to the first two top layers of $\mathrm{Pt}_{25} \mathrm{Rh}_{75}$, and $\mathrm{Pt}$ segregation to the top layer of $\mathrm{Pt}_{50} \mathrm{Rh}_{50}$, which can also be predicted by the on-site energy only. On the contrary, $\mathrm{Pt}$ composition of the second layer in $\mathrm{Pt}_{50} \mathrm{Rh}_{50}$ exhibits a minimum around $T=300 \mathrm{~K}$ in the full-ECI calcu- lation. This can be attributed to the occurrence of the layerconfined order-disorder transition between 200 and $250 \mathrm{~K}$. The simulated ordered structure is designated as $(\sqrt{3}$ $\times \sqrt{3}) R 30^{\circ}$ in a composition of $\mathrm{Pt}_{33.3} \mathrm{Rh}_{66.7}$, which is a superlattice along the intralayer [211] direction. Since this ordered structure has not been reported experimentally, accurate measurements of ordering tendency for the Pt-Rh (111) surface are highly desirable.

Note added. Recently, a DFT-based study of surface segregation, relaxation, and ordering for the $\mathrm{Pt}_{25} \mathrm{Rh}_{75}$ (111) surface by Müller et al. [Appl. Phys. A 82, 415 (2006)] was brought to our attention. Using a combination of the cluster expansion technique and Monte Carlo simulation, they calculated the surface segregation profile, which is similar to those shown in the present study. Some differences between their work and the present work may come from differences in computational details. Although these studies address similar subjects, the major points of the discussion are different: They discuss the segregation profile with a focus on atomic structure, and also SRO parameters at the top layer. Meanwhile, we focus on the contribution of the mixing energy and lattice vibration to the segregation, and discuss atomic ordering in the surface, comparing with bulk ordered structure.

\section{ACKNOWLEDGMENTS}

This work was financially supported by the New Energy and Industrial Technology Development Organization (NEDO) of Japan.

\section{APPENDIX A}

The flipping probability $P$ of Eq. (13) including the vibrational effects is derived here. Let us start from a partition function of a system, given by

$$
Z=\sum_{s \in \text { states }} \exp \left(-\frac{E_{s}}{k_{B} T}\right)
$$

In the present article, we have considered the configurational and vibrational contributions to the partition function, and all the other contributions have been neglected. Since the lifetime of a particular configuration $\gamma$ should be long enough to achieve vibrational equilibrium, ${ }^{44}$ summation of state $s$ can be independently performed for configurational and vibrational contribution, namely,

$$
Z \simeq \sum_{\gamma}\left[\exp \left(-\frac{E_{\text {stat }}(\gamma)}{k_{B} T}\right) \sum_{v} \exp \left(-\frac{E_{\mathrm{vib}}^{v}(\gamma)}{k_{B} T}\right)\right],
$$

where $E_{\text {stat }}(\gamma)$ denotes a static contribution to the internal energy at each configuration $\gamma$, and $E_{\mathrm{vib}}^{v}(\gamma)$ is the vibrational contribution of state $v$ at each configuration $\gamma$. Thus the vibrational partition function can be given for each $\gamma$,

$$
Z_{\mathrm{vib}}(\gamma)=\sum_{v} \exp \left(-\frac{E_{\mathrm{vib}}^{v}(\gamma)}{k_{B} T}\right)
$$


Now we can describe corresponding vibrational free energy as

$$
F_{\text {vib }}(\gamma)=-k_{B} T \ln Z_{\text {vib }}(\gamma) .
$$

The vibrational free energy $F_{\text {vib }}(\gamma)$ depends only on temperature $T$ and configuration $\gamma$, which is similar to the expression of Eq. (8). Therefore, we can finally rewrite the partition function $Z$ of Eq. (A2) as

$$
Z=\sum_{\gamma} \exp \left(-\frac{E_{\text {stat }}(\gamma)+F_{\mathrm{vib}}(\gamma)}{k_{B} T}\right)
$$

It is clear from Eq. (A5) that for MC simulations of configurational thermodynamics, a canonical ensemble can be actualized by sampling each state with its weight to be $\exp \left\{-\left[E_{\text {stat }}(\gamma)+F_{\text {vib }}(\gamma)\right] / k_{B} T\right\}$. This immediately yields the flipping probability of a trial state, given by Eq. (13).
${ }^{1}$ R. M. Wolf, J. Siera, F. C. M. J. M. van Delft, and B. E. Nieuwenhuys, Faraday Discuss. Chem. Soc. 87, 275 (1989).

${ }^{2}$ T. B. Massalski, Binary Alloy Phase Diagrams (ASM International, Materials Park, OH, 1990).

${ }^{3}$ E. Raub, J. Less-Common Met. 1, 3 (1959).

${ }^{4}$ E. Raub and G. Falkenburg, Z. Metallkd. 55, 392 (1964).

${ }^{5}$ V. E. Antonov, T. E. Antonova, I. T. Belash, E. G. Ponyatovshii, and V. I. Rashupkin, Phys. Status Solidi A 78, 137 (1983).

${ }^{6}$ S. R. Bharadwaj, A. S. Kerkar, S. N. Tripathi, and S. R. Dharwadkar, J. Less-Common Met. 169, 167 (1991).

${ }^{7}$ Z. W. Lu, B. M. Klein, and A. Zunger, J. Phase Equilib. 16, 36 (1995).

${ }^{8}$ Ch. Steiner, B. Schönfeld, M. J. Portmann, M. Kompatscher, G. Kostorz, A. Mazuelas, T. Metzger, J. Kohlbrecher, and B. Deme, Phys. Rev. B 71, 104204 (2005).

${ }^{9}$ L. Anthony, J. K. Okamoto, and B. Fultz, Phys. Rev. Lett. 70, 1128 (1993).

${ }^{10}$ L. Anthony, L. J. Nagel, J. K. Okamoto, and B. Fultz, Phys. Rev. Lett. 73, 3034 (1994).

${ }^{11}$ B. Fultz, L. Anthony, L. J. Nagel, R. M. Nicklow, and S. Spooner, Phys. Rev. B 52, 3315 (1995).

${ }^{12}$ B. Fultz, L. Anthony, J. L. Robertson, R. M. Nicklow, S. Spooner, and M. Mostoller, Phys. Rev. B 52, 3280 (1995).

${ }^{13}$ G. D. Garbulsky and G. Ceder, Phys. Rev. B 53, 8993 (1996).

${ }^{14}$ A. van de Walle, G. Ceder, and U. V. Waghmare, Phys. Rev. Lett. 80, 4911 (1998).

${ }^{15}$ V. Ozolins, C. Wolverton, and A. Zunger, Phys. Rev. B 58, R5897 (1998).

${ }^{16}$ C. Wolverton and V. Ozolins, Phys. Rev. Lett. 86, 5518 (2001).

${ }^{17}$ I. A. Abrikosov, A. V. Ruban, H. L. Skriver, and B. Johansson, Phys. Rev. B 50, R2039 (1994).

${ }^{18}$ F. L. Williams and G. C. Nelson, Appl. Surf. Sci. 3, 409 (1979).

${ }^{19}$ P. H. Holloway and F. L. Williams, Appl. Surf. Sci. 10, 1 (1982).

${ }^{20}$ F. C. M. J. M. van Delft and B. E. Nieuwenhuys, Surf. Sci. 162, 538 (1985).

${ }^{21}$ A. D. van Langeveld and J. W. Niementsverdriet, Surf. Sci. 178, 880 (1986).

${ }^{22}$ D. M. Ren and T. T. Tsong, Surf. Sci. 184, L439 (1987).

${ }^{23}$ T. T. Tsong, D. M. Ren, and M. Ahmad, Phys. Rev. B 38, 7428 (1988).

${ }^{24}$ P. Varga and M. Schmid, Appl. Surf. Sci. 141, 287 (1999).

${ }^{25}$ E. Platzgummer, M. Sporn, R. Koller, S. Forsthuber, M. Schmid, W. Hofer, and P. Varga, Surf. Sci. 419, 236 (1999).

${ }^{26}$ E. L. D. Hebenstreit, W. Hebenstreit, M. Schmid, and P. Varga, Surf. Sci. 441, 441 (1999).

${ }^{27}$ D. Brown, P. D. Quinn, D. P. Woodruff, T. C. Q. Noakes, and P. Bailey, Surf. Sci. 497, 1 (2002).
${ }^{28}$ B. Legrand and G. Tréglia, Surf. Sci. 236, 398 (1990).

${ }^{29}$ J. Florencio, D. M. Ren, and T. T. Tsong, Surf. Sci. 345, L29 (1996).

${ }^{30}$ L. Z. Mezey and W. Hofer, Surf. Sci. 402-404, 845 (1998).

${ }^{31}$ A. V. Ruban and H. L. Skriver, Comput. Mater. Sci. 15, 119 (1999).

${ }^{32}$ L. V. Pourovskii, A. V. Ruban, I. A. Abrikosov, Y. Kh. Vekilov, and B. Johansson, Phys. Rev. B 64, 035421 (2001).

${ }^{33}$ G. Kresse and J. Hafner, Phys. Rev. B 47, R558 (1993).

${ }^{34}$ G. Kresse and J. Furthmüller, Phys. Rev. B 54, 11169 (1996).

${ }^{35}$ G. Kresse and D. Joubert, Phys. Rev. B 59, 1758 (1999).

${ }^{36}$ P. E. Blöchl, Phys. Rev. B 50, 17953 (1994).

${ }^{37}$ J. P. Perdew and Y. Wang, Phys. Rev. B 45, 13244 (1992).

${ }^{38}$ M. Methfessel and A. T. Paxton, Phys. Rev. B 40, 3616 (1989).

${ }^{39}$ H. J. Monkhost and J. D. Pack, Phys. Rev. B 13, 5188 (1976).

${ }^{40}$ C. Kittel, Introduction to Solid State Physics, 6th ed. (Wiley, New York, 1986), p. 25.

${ }^{41}$ K. Parlinski, Z. Q. Li, and Y. Kawazoe, Phys. Rev. Lett. 78, 4063 (1997).

${ }^{42}$ R. P. Feynman, Phys. Rev. 56, 340 (1939).

${ }^{43}$ J. M. Sanchez, F. Ducastelle, and D. Gratias, Physica A 128A, 334 (1984)

${ }^{44}$ D. de Fountain, Solid State Physics, edited by H. Ehrenreich and D. Turnbull (Academic Press, Cambridge, Massachusetts, 1994), Vol. 47, pp. 33-176.

${ }^{45}$ Stefan Müller, J. Phys.: Condens. Matter 15, R1429 (2003).

${ }^{46}$ Z. W. Lu, S.-H. Wei, A. Zunger, S. Frota-Pessoa, and L. G. Ferreira, Phys. Rev. B 44, 512 (1991).

${ }^{47}$ M. Stone, J. R. Stat. Soc. Ser. B (Methodol.) 36, 111 (1974).

${ }^{48}$ D. M. Allen, Technometrics 16, 125 (1974).

${ }^{49}$ A. van de Walle and G. Ceder, Rev. Mod. Phys. 74, 11 (2002).

${ }^{50}$ K. Yuge, A. Seko, I. Tanaka, and S. R. Nishitani, Phys. Rev. B 72, 174201 (2005).

${ }^{51}$ Y. S. Touloukian, R. K. Kirby, R. E. Taylor, and P. D. Desai, Thermophysical Properties of Matter (IFI/PLENUM, New York, 1976), Vol. 12, pp. 156-164.

${ }^{52}$ A. A. Maradudin, E. W. Montroll, G. H. Weiss, and I. P. Ipatova, Theory of Lattice Dynamics in the Harmonic Approximation, 2nd ed. (Academic, New York, 1971).

${ }^{53}$ A. van de Walle, Ph.D. thesis, Massachusetts Institute of Technology (2000)

${ }^{54}$ N. Metropolis, A. W. Rosenbluth, M. N. Rosenbluth, A. H. Teller, J. Chem. Phys. 21, 1087 (1953).

${ }^{55}$ K. S. Kirkpatrick, C. D. Gelatt, and M. P. Vecchi, Science 220, 671 (1983)

${ }^{56}$ K. S. Kirkpatrick, J. Stat. Phys. 34, 975 (1984).

${ }^{57}$ S. M. Foiles, Phys. Rev. B 32, 7685 (1985). 
${ }^{58}$ A. Seki, D. N. Seidman, Y. Oh, and S. M. Foiles, Acta Metall. Mater. 39, 3167 (1991).

${ }^{59}$ D. Udler and D. N. Seidman, Acta Metall. Mater. 42, 1959 (1994).

${ }^{60}$ A. Z. Panagiotopoulos, Mol. Phys. 62, 701 (1987).

${ }^{61}$ S. Curtarolo, D. Morgan, and G. Ceder, CALPHAD: Comput. Coupling Phase Diagrams Thermochem. 29, 163 (2005).

${ }^{62}$ K. T. Jacob, S. Priya, and Y. Waseda, Metall. Mater. Trans. A 29, 1545 (1998).

${ }^{63}$ J. M. Cowley, J. Appl. Phys. 21, 24 (1950).
${ }^{64}$ H. L. Skriver and N. M. Rosengaard, Phys. Rev. B 46, 7157 (1992).

${ }^{65}$ L. Vitos, A. V. Ruban, H. L. Skriver, and J. Kollá, Surf. Sci. 411, 186 (1998).

${ }^{66}$ I. Galanakis, N. Papanikolaou, and P. H. Dederichs, Surf. Sci. 511, 1 (2002).

${ }^{67}$ F. R. de Boer, R. Boom, W. C. M. Mattens, A. R. Miedema, and A. K. Niessen, Cohesion in Metals (North-Holland, Amsterdam, 1988). 